\title{
Ultrasensitive hyperspectral imaging and biodetection enabled by dielectric metasurfaces
}

\author{
Filiz Yesilkoy ${ }^{1}$, Eduardo R. Arvelo ${ }^{1,2}$, Yasaman Jahani ${ }^{1}$, Mingkai Liu ${ }^{3}$, Andreas Tittl ${ }^{1}$, \\ Volkan Cevher ${ }^{2}$, Yuri Kivshar ${ }^{3}$, and Hatice Altug ${ }^{1 *}$
}

\footnotetext{
${ }^{1}$ Interfaculty Institute of Bioengineering, École polytechnique fédérale de Lausanne (EPFL), Lausanne 1015, Switzerland.

${ }^{2}$ Electrical Engineering Institute, École polytechnique fédérale de Lausanne (EPFL), Lausanne 1015, Switzerland.

${ }^{3}$ Nonlinear Physics Centre, Australian National University, Canberra, ACT 2601, Australia.
}

Metasurfaces based on resonant subwavelength photonic structures enable novel ways of wavefront control and light focusing, underpinning a new generation of flat-optics devices ${ }^{1}$. Recently emerged all-dielectric asymmetric metasurfaces, composed of arrays of meta-units with broken in-plane inversion symmetry ${ }^{2-7}$, exhibit high-quality resonances originating from the intriguing physics of bound states in the continuum. Here, we combine dielectric metasurfaces and hyperspectral imaging to develop an ultrasensitive label-free analytical platform for biosensing. Our technique can acquire spatially resolved spectra from millions of image pixels and use smart data processing tools to extract high-throughput digital sensing information at the unprecedented level of less than three molecules per $\boldsymbol{\mu m}^{2}$. We further show spectral data retrieval from a single image without using spectrometers, enabled by our unique sensor design, paving the way for portable diagnostic applications. This combination of nanophotonics and imaging optics extends capabilities of dielectric metasurfaces to analyse biological entities and atomic layer thick two-dimensional materials over large areas.

Bound states in the continuum (BIC) were discovered in quantum mechanics ${ }^{8}$, but were later employed as the important physical concept of destructive interference ${ }^{9}$, expanding to other fields of wave physics, including optics ${ }^{10,11}$. In practice, BIC can be realized as a quasi-BIC in the form of a supercavity mode ${ }^{12}$ when both quality-factor $(\mathrm{Q})$ and resonance width become finite at the BIC conditions due to absorption and other perturbations ${ }^{13}$. Particularly, in contrast to their plasmonic counterparts, dielectric nanoresonators do not suffer from inherent material absorption losses ${ }^{14,15}$ and they can support BIC modes generating extremely sharp resonances and strong light confinement. Importantly, dielectric metasurfaces that exhibit these supercavity modes in visible and near infrared (NIR) wavelengths can be extremely responsive to the local refractive index (RI) changes induced by individual biomolecules, due to their spatial overlap with the surface-confined electromagnetic fields ${ }^{16}$. This is in contrast to conventional Mie-type resonances in silicon subwavelength nanoparticles ${ }^{15}$, which store the field mainly inside the high-index material. Previously, silicon nanostructures with Mie-type resonances in NIR were explored for label-free biosensing ${ }^{17,18}$, where traditional spectrometers were used to monitor resonance shifts from spatially limited regions and to quantify analyte mass accumulation using ensemble averaging. The exploration of the BIC-inspired high-Q modes of dielectric metasurfaces in conjunction with imaging-based, high-content data 
acquisition and processing has yet to be leveraged to launch nanophotonic biosensors as a game changer in the biomedical sciences.

Here, we propose and demonstrate a novel platform based on all-dielectric metasurfaces, supporting geometrically tunable high-Q BIC-inspired resonances in the NIR, and hyperspectral imaging (HSI) for massively parallel sensing of trace biomolecules as well as optical characterisation of two-dimensional (2D)-materials at their relevant atomic dimension scale. Our HSI platform spatially resolves spectral information, generating millions of spectra, each associated to a complementary metal oxide semiconductor sensor (CMOS) pixel, without using spectrometers or mechanical scanning. This is particularly important to achieve extreme sensitivities and throughput via the use of various data science techniques. We demonstrate that by employing a pixel-based thresholding method the detection limit can be improved by three orders of magnitude when compared to ensemble averaging ( $\sim 3$ vs 1500 molecules $\left./ \mu \mathrm{m}^{2}\right)$. Moreover, we introduce a multi resonance sensor design and the new concept of a hyperspectral decoder, which associates spectral information from each CMOS pixel to its spatial index. With the use of this decoder, we demonstrate that high-resolution spectral data can be retrieved from a single image captured at a fixed wavelength. This scheme eliminates the need for bulky and expensive instrumentation and can be used to realize field-deployable sensors. Finally, we subject our sensor to a single atomic layer of graphene and show that our approach is capable of efficiently probing graphene's optical properties over large areas.

Our nanophotonic device comprises an array of all-dielectric sensors allowing for multiplexed analyte detection in a single measurement. In our optical setup, we use a continuously tunable bandpass filter coupled to a supercontinuum laser source for excitation in a robust collinear optical path and record images at each illumination wavelength using a CMOS camera (Fig. 1a). This optical configuration allows us to spatially probe the metasurface spectral response over large areas, contrary to traditional spectrometers ${ }^{19}$. Specifically, we use an extremely narrow bandwidth $(\sim 2 \mathrm{~nm})$ and tunable excitation with high spectral resolution $(\Delta \lambda=0.1 \mathrm{~nm})$ to build a hyperspectral data cube of the field-of-view that contains full spectral information from the entire sensor array, where each sensor is mapped by tens of thousands of CMOS pixels. The high-content data generated by this method allows for unprecedented use of data science techniques to best extract insights and information from each sensor ${ }^{20}$. For example, the data cube can be processed to create a resonance map for each sensor (Fig. 1b), where an individual image pixel represents an area on the metasurface and its value corresponds to the centre wavelength of the area's resonance. This is particularly important for biosensing, because resonance shifts caused by the presence of analytes on the sensor's surface are spatially registered by the resonance maps. These maps are then compared with reference maps, acquired without the analyte, to compute resonance shift maps, where each pixel conveys spectral shift information of its corresponding area on the sensor, as shown in Fig. $1 \mathrm{~b}$.

Our resonant metasurface consists of pairs of tilted silicon nanobars (see Figs. 2a-f), which are fabricated using CMOS compatible processes. Fig. 2c shows a photograph of a dielectric chip with a 5 x 5 sensor array, manufactured by nanostructuring a $100 \mathrm{~nm}$ thick amorphous Si film on a glass substrate, followed by dry etching (Fig. S1). Scanning electron microscopy (SEM) images of a metaunit and metasurface are shown in Figs. 2a, b, respectively. In our metasurface 
design, the elliptical Si pairs of nanobars are tilted around the y-axis in mirror symmetry (Fig. 2d). Normally incident x-polarized light excites the collective lattice resonances, which have their origin in the BIC-inspired physics ${ }^{2}$, where sharp spectral resonances are controlled by the asymmetry parameter introduced by the tilting angle, such that the Q-factor grows to infinity when the tilting angle vanishes. In our design, we leverage the quasi-BIC states, which are achieved with small but finite values of the tilting angle $\left(17.5^{\circ}\right)$, leading to high-Q and spectrally isolated resonances with high suppression (see Fig. S2). The resulting strong light trapping occurs in such a way that both electric and magnetic fields are localized in the surrounding outer volume of the nanostructures (Fig. 2f), ideal for sensing applications (see Fig. S3).

More importantly, our dielectric metasurface can be spectrally tuned by geometrically scaling the resonator dimensions via the parameter $\alpha$, as shown in Fig. $2 \mathrm{~g}$ (see also Fig. S4), where the transmission spectra are extracted from single CMOS pixels, each representing a sensor area of $0.5 \mu \mathrm{m}^{2}$, in the presence of a top medium with $\mathrm{n}_{\mathrm{M}}=1.47$. The measured sharp resonances in the near-IR are similar to the spectra obtained using conventional spectrometers (see Fig. S5). To study the Q-factor of the resonance mode, the full width at half maximum (FWHM) of the resonance dip is measured with numerous concentrations of glycerol solutions $\left(\mathrm{n}_{\mathrm{M}}=1: 1.47\right)$. The measured FWHM results are plotted in Fig. 2h, showing Q-factors as high as 144, which is a significant achievement for all-dielectric planar metasurfaces in the CMOS detectable spectral range. For more details on the importance of high-Q resonances for sensing small spectral shifts induced by single molecules, see Figs. S2 and S6. Furthermore, our dielectric resonators attain high near-field enhancement (40-times), which results in a refractometric sensitivity of $263 \mathrm{~nm} / \mathrm{RIU}$ (see Fig. S3).

To demonstrate the compatibility of all-dielectric metasurfaces with biorecognition assays, we use epoxy-silane chemistry to covalently immobilize the capture molecules onto the sensor surface $^{21}$. Mouse-derived Immunoglobulin $\mathrm{G}(\mathrm{M}-\mathrm{IgG})$ solutions were first selectively deposited on the sensor arrays using a low-volume liquid dispenser (Figs. 3a, c), where areal molecular density on a sensor is controlled by adjusting the droplet volume and the analyte concentration. For control sensors, we deposited bovine serum albumin (BSA) instead of MIgG. After incubation, the remaining chip surface was blocked with BSA to minimize nonspecific binding. Next, the sensors were incubated with rabbit-derived anti-mouse IgG (R$\mathrm{IgG}$ ), which has high affinity to the $\mathrm{M}-\mathrm{IgG}$, ensuring that surface-immobilized $\mathrm{M}-\mathrm{IgG}$ molecules bind to R-IgG (Fig. 3b). This model bioassay enables the quantification of areal molecular density, as well as the determination of the detection limit of the metasurface, independent of the bioassay affinity parameters (see Fig. S7).

To evaluate the biosensing performance of our system, we compute the resonance shift maps from sensors with different analyte quantities $(\mathrm{N}=66)$, a subset of which is shown in Fig. $3 \mathrm{~g}$. A traditional calibration curve that correlates the ensemble-averaged resonance shift over the analyte spot to the average number of analyte molecules per area is shown in Fig. 3d, where the colour bars indicate the data points associated to the resonance shift maps shown in Fig. $3 \mathrm{~g}$. The measurements with low areal molecule density $\left(<10^{3}\right.$ molecules $\left./ \mu \mathrm{m}^{2}\right)$ are not detectable by the traditional ensemble-average method, however the analyte spots are visible 
on the resonance shift maps. In fact, this is a major challenge that analytical biosensing platforms face, as the signal from ensemble measurements is masked by the background noise at low concentrations ${ }^{22}$.

In contrast, our imaging-based platform allows for digitized biomolecular detection, as enhanced analytical characterization is possible by evaluating the information carried by each individual pixel. Due to the highly localized fields of the metasurface, when a few molecules attach to the so-called "hotspots," a significant spectral shift occurs (see Fig. S8). Yet, as the total number of molecules on the sensor decrease, so does the number of metaunits that have enough molecules to induce a measurable shift due to the stochastic nature of the binding event. Therefore, we use a thresholding method to classify whether a resonance shift map pixel belongs to the region where the analyte is present. The associated receiver operating characteristic (ROC) curves, attained by varying the threshold value, from a sample subset of the data presented in Fig. $3 \mathrm{~d}$ are shown in Fig. $3 \mathrm{f}$ (see also Fig. S7). To quantify the signal, we use the metric of the area under the ROC curve (AUC) ${ }^{23}$, which is expected to equal 1 when the perfect separation point is achieved and 0.5 for control measurements. Fig. 3e shows the AUC values for the same dataset used in Fig. 3d. The baseline was computed using the standard criterion of three standard deviations above the mean value of control measurements $(0.5008$ $\pm 0.0291, \mathrm{~N}=68)$. This approach reveals biomolecular detection information at very low molecular counts $\left(\sim 3\right.$ molecules $\left./ \mu \mathrm{m}^{2}\right)$, corroborating the strength of our label-free imaging method (see Fig. S9).

The tunable and sharp resonant modes exhibited by our metasurfaces enable the design of unique multi-resonance sensors (MRS) for spectrometerless retrieval of spectral information from a single image acquired at a fixed wavelength, which allows for potential miniaturization of our system. Fig. 4a shows the resonance map of a $200 \mu \mathrm{m}$ x $200 \mu \mathrm{m}$ MRS, which is spatially partitioned into sixteen sub-sensors by varying the tuning parameter as described in Fig. $4 \mathrm{~b}$. Each MRS is then hyperspectrally imaged by $275 \times 275$ CMOS pixels with water as top medium. The different tuning parameters, aided by intrinsic fabrication variances ${ }^{24}$, yield sensors with a wide range of densely distributed resonance wavelengths that respond to changes in the top media refractive index identically. Moreover, since the footprint of an individual MRS is small, sensor arrays over large imaging areas can be imaged in a single snapshot for multiplexed detection (see Figs. S10 and S11).

To translate the intensity data from a single image into spectral information, it is necessary to compute an index decoder using the resonance map. We begin by stacking the resonance map of MRS column-wise as shown in Fig. 4c, where each pixel is indexed from 1 to $275^{2}$. Rearranging this resonance wavelength vector in increasing order yields a sorted resonance vector and an associated index decoder, which contains the order that the pixels must be arranged to achieve the sorting (Fig. 4d). The index decoders are unique signatures of the sensors and can be used to implement a barcode-based sensing scheme. To illustrate this procedure, three images of the same sensor with different top media (water, $5 \%$ and $10 \%$ glycerol) are acquired at the single wavelength of $824 \mathrm{~nm}$ (Fig. 4e). These images are then stacked to reveal the sensing barcodes (Fig. 4f), which are unique to the optical properties of the sensor and the refractive index of the top medium. The sensing barcodes are decoded by 
rearranging their elements according to the previously computed index decoder and binning the intensity values to achieve uniform wavelength representation (Fig. 4g). The resulting vector is a pseudo-spectrum that can be interpreted for spectral shift sensing, as shown in Fig. 4h. In this example, since the index decoder is computed from reference data acquired with water medium, the pixels that resonate at the probing wavelength $(824 \mathrm{~nm})$ are the darkest for the case with water as top medium. When the sensor is imaged with a top medium of higher refractive index, some of the pixels whose resonance wavelengths are initially less than 824 $\mathrm{nm}$ become resonant due to the red shift of the resonance dip. The difference in the resonance wavelength of such pixels corresponds to the spectral shift associated with the difference in the refractive index of the top media. This novel technique is particularly important for portable on-site sensing platforms, where an off-the-shelf single wavelength source, coupled with a CMOS camera and a single-board computer can be used to build a hand-held barcode reader.

To investigate the near-field probing capabilities of our novel sensing platform, we measured the spectral changes induced by a single-layer graphene (SLG) covering the metasurface (Fig. 5a). The graphene was characterized with Raman spectroscopy to ensure the transfer was in fact of single-layer quality (See Fig. S12 and Table S1) ${ }^{25,26}$. Hyperspectrally measured and simulation extracted transmission spectra with and without $\sim 3.2 \AA$-thick SLG on the dielectric metasurface are shown in Fig. 5b. Both simulation and experimental results indicate a remarkable $\sim 3 \mathrm{~nm}$ red-shift and linewidth broadening in the resonance dip due to SLG's optical properties in near-IR $(\mathrm{n}=2.69, \mathrm{k}=1.52)^{27}$. When hyperspectral data cubes are used, our nanophotonic imaging setup can spatially resolve the optical properties of SLG over large areas $\left(3.3 \mathrm{~mm}^{2}\right)$, as shown in Figs. $5 \mathrm{c}$ and $5 \mathrm{~d}$. Moreover, our single image-based barcode sensing technique can also be utilized to retrieve the spectral changes that emerge due to the enhanced localized interaction of the metasurface with graphene. Fig. 5e shows the single-wavelength intensity images of MRS with and without SLG along with their corresponding sensing barcodes. Once the index decoder (Fig. 5f) is used to rearrange the corresponding sensing barcodes, spectral sensing information can be retrieved (Fig. 5g). Importantly, both the spectral shift and the line-broadening information is recovered in agreement with the hyperspectral results shown in Fig. 5b. This demonstrates that our unique method can efficiently characterize the optical parameters of SLG over large areas from a single CMOS image without the need of a spectrometer, which is crucial for the current efforts in understanding and engineering lowdimensional materials ${ }^{28}$.

We have demonstrated novel applications of high-Q resonant dielectric metasurfaces that support bound states in the continuum, in conjunction with advanced imaging-based optical data acquisition and processing methods, to build a superior and versatile sensing platform. Alternative materials and designs that support such resonant modes can be explored to further enhance the sensitivity and the Q-factor of the resonant metasurfaces. Moreover, the spectral range and density of our single-wavelength spectral retrieval method can be increased by exploiting actively tunable materials or by probing unutilized dimensions, such as the polarization of the incident light. Finally, machine learning and artificial intelligence methods can introduce new inference tools to expedite our platform as a field-deployable highthroughput single-molecule detector for biomedical applications. 


\section{References}

1. Capasso, F. The future and promise of flat optics: a personal perspective. Nanophotonics 7, 953-957 (2018).

2. Campione, S. et al. Broken symmetry dielectric resonators for high quality factor fano metasurfaces. ACS Photonics 3, 2362-2367 (2016).

3. Liu, M., Powell, D. A., Guo, R., Shadrivov, I. V. \& Kivshar, Y. S. Polarization-Induced Chirality in Metamaterials via Optomechanical Interaction. Advanced Optical Materials 5, 1600760 (2017).

4. Tuz, V. R. et al. High-quality trapped modes in all-dielectric metamaterials. Opt. Express, OE 26, 2905-2916 (2018).

5. Tittl, A. et al. Imaging-based molecular barcoding with pixelated dielectric metasurfaces. Science 360, 1105-1109 (2018).

6. Koshelev, K., Lepeshov, S., Liu, M., Bogdanov, A. \& Kivshar, Y. Asymmetric metasurfaces with high- $\$ Q \$$ resonances governed by bound states in the continuum. Phys. Rev. Lett. 121, 193903 (2018).

7. Koshelev, K., Bogdanov, A., \& Kivshar, Y., Meta-optics and bound states in the continuum, Science Bulletin (2018), doi: https://doi.org/10.1016/j.scib.2018.12.003.

8. von Neuman, J. \& Wigner, E. Uber merkwürdige diskrete Eigenwerte. Uber das Verhalten von Eigenwerten bei adiabatischen Prozessen. Physikalische Zeitschrift 30, 467-470 (1929).

9. Friedrich, H. \& Wintgen, D. Interfering resonances and bound states in the continuum. Phys. Rev. A 32, 3231-3242 (1985).

10. Marinica, D. C., Borisov, A. G. \& Shabanov, S. V. Bound states in the continuum in photonics. Phys. Rev. Lett. 100, 183902 (2008).

11. Bulgakov, E. N. \& Sadreev, A. F. Bound states in the continuum in photonic waveguides inspired by defects. Phys. Rev. B 78, 075105 (2008). 
12. Rybin, M. \& Kivshar, Y. Optical physics: Supercavity lasing. Nature 541, 164-165 (2017).

13. Doeleman, H. M., Monticone, F., den Hollander, W., Alù, A. \& Koenderink, A. F. Experimental observation of a polarization vortex at an optical bound state in the continuum. Nature Photonics 12, 397-401 (2018).

14. Kruk, S. \& Kivshar, Y. Functional meta-optics and nanophotonics governed by Mie resonances. ACS Photonics 4, 2638-2649 (2017).

15. Kuznetsov, A. I., Miroshnichenko, A. E., Brongersma, M. L., Kivshar, Y. S. \& Luk'yanchuk, B. Optically resonant dielectric nanostructures. Science 354, aag2472 (2016).

16. Fabrizio, E. D. et al. Roadmap on biosensing and photonics with advanced nano-optical methods. J. Opt. 18, 063003 (2016).

17. Bontempi, N. et al. Highly sensitive biosensors based on all-dielectric nanoresonators. Nanoscale 9, 4972-4980 (2017).

18. Yavas, O., Svedendahl, M., Dobosz, P., Sanz, V. \& Quidant, R. On-a-chip biosensing based on all-dielectric nanoresonators. Nano Letters 17, 4421-4426 (2017).

19. Lee, S. H., Lindquist, N. C., Wittenberg, N. J., Jordan, L. R. \& Oh, S.-H. Real-time fullspectral imaging and affinity measurements from 50 microfluidic channels using nanohole surface plasmon resonance. Lab Chip 12, 3882-3890 (2012).

20. Ballard, Z. S. et al. Computational Sensing Using Low-Cost and Mobile Plasmonic Readers Designed by Machine Learning. ACS Nano 11, 2266-2274 (2017).

21. Tsukruk, V. V., Luzinov, I. \& Julthongpiput, D. Sticky Molecular Surfaces: Epoxysilane Self-Assembled Monolayers. Langmuir 15, 3029-3032 (1999).

22. Walt, D. R. Optical methods for single molecule detection and analysis. Anal. Chem. $\mathbf{8 5}$, $1258-1263$ (2013).

23. Bradley, A. P. The use of the area under the ROC curve in the evaluation of machine learning algorithms. Pattern Recognition 30, 1145-1159 (1997). 
24. Karst, J., Strohfeldt, N., Schäferling, M., Giessen, H. \& Hentschel, M. Single plasmonic oligomer chiral spectroscopy. Advanced Optical Materials 6, 1800087 (2018).

25. Ferrari, A. C. \& Basko, D. M. Raman spectroscopy as a versatile tool for studying the properties of graphene. Nature Nanotechnology 8, 235-246 (2013).

26. Ni, Z. H. et al. Probing Charged Impurities in Suspended Graphene Using Raman Spectroscopy. ACS Nano 3, 569-574 (2009).

27. Ghamsari, B. G., Tosado, J., Yamamoto, M., Fuhrer, M. S. \& Anlage, S. M. Measuring the complex optical conductivity of graphene by fabry-pérot reflectance spectroscopy. Scientific Reports 6, (2016).

28. Ogletree, D. F. et al. Revealing optical properties of reduced-dimensionality materials at relevant length scales. Advanced Materials 27, 5693-5719

\section{Acknowledgements}

We thank D.N. Neshev, A. Avsar and A. Belushkin for fruitful discussions, Y. Pandey and S. Confederat for assistance in preparing the sensor chips, A. Magrez for assistance with Raman spectroscopy, École polytechnique fédérale de Lausanne and Center of MicroNano Technology for nanofabrication. The research leading to these results has received funding from the European Research Council under grant agreement no. 682167 VIBRANT-BIO and the European Union Horizon 2020 Framework Programme for Research and Innovation under grant agreements no. 665667 (call 2015), no. 777714 (NOCTURNO project), no. FETOPEN737071 (ULTRACHIRAL project), and no. 644956 (RAIS project). YK acknowledges support of the Strategic Fund of the Australian National University.

\section{Author contributions}

F.Y., E.R.A, Y.S.K., and H.A. conceived and designed the research; F.Y. and Y.J. fabricated the dielectric metasurfaces; F.Y. and E.R.A. carried out optical measurements; F.Y., E.R.A, and V.C. analysed data; F.Y., Y.J., A.T., and M.L. carried out numerical simulations; and all authors contributed to writing the manuscript.

\section{Competing interests}

None declared.

\section{Additional information}

Supplementary information is available for this paper

Correspondence and requests for materials should be addressed to H.A. 


\section{Figures}

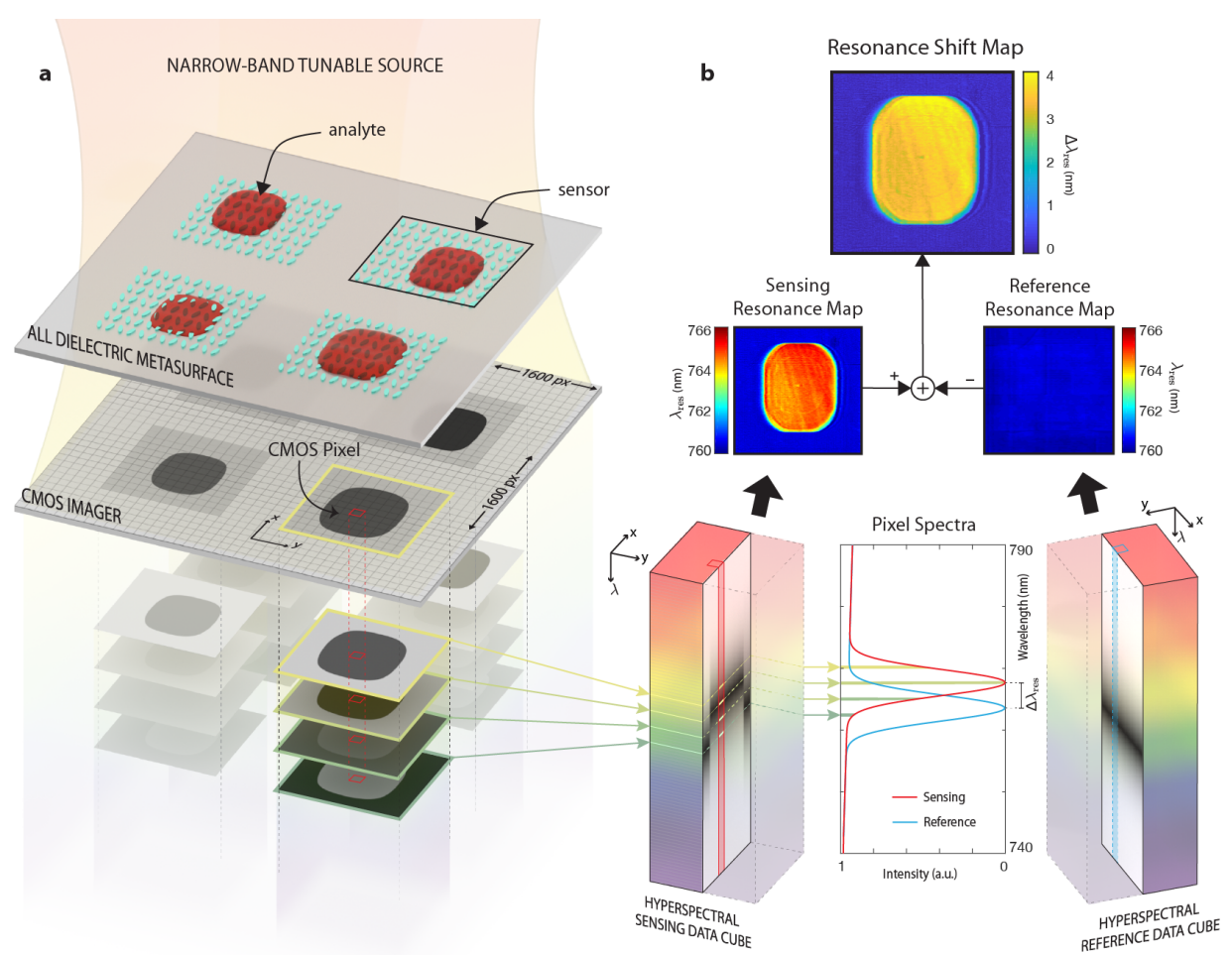

Figure 1 | Principle of hyperspectral imaging-based biomolecule detection using all-dielectric metasurfaces. (a) Sketch of the hyperspectral imaging principle showing a representative dielectric metasurface sensor array illuminated with narrow-band tunable laser source. At each wavelength illumination, images are recorded by a CMOS camera (1608x1608 pixels) to create a hyperspectral data cube where each CMOS pixel captures high resolution spectral information. (b) The hyperspectral data cube is processed to extract spatial resonance maps of each individual sensor. For biomolecule detection, the sensing and reference resonance maps are combined to create the resonance shift map, which conveys spectral shift information across the whole sensor. 


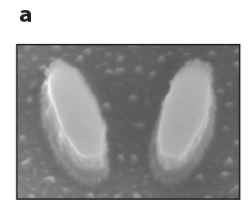

$\longmapsto 100 \mathrm{~nm}$

b
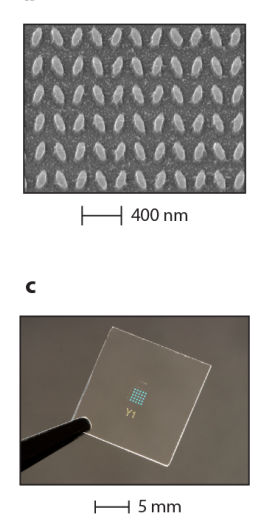
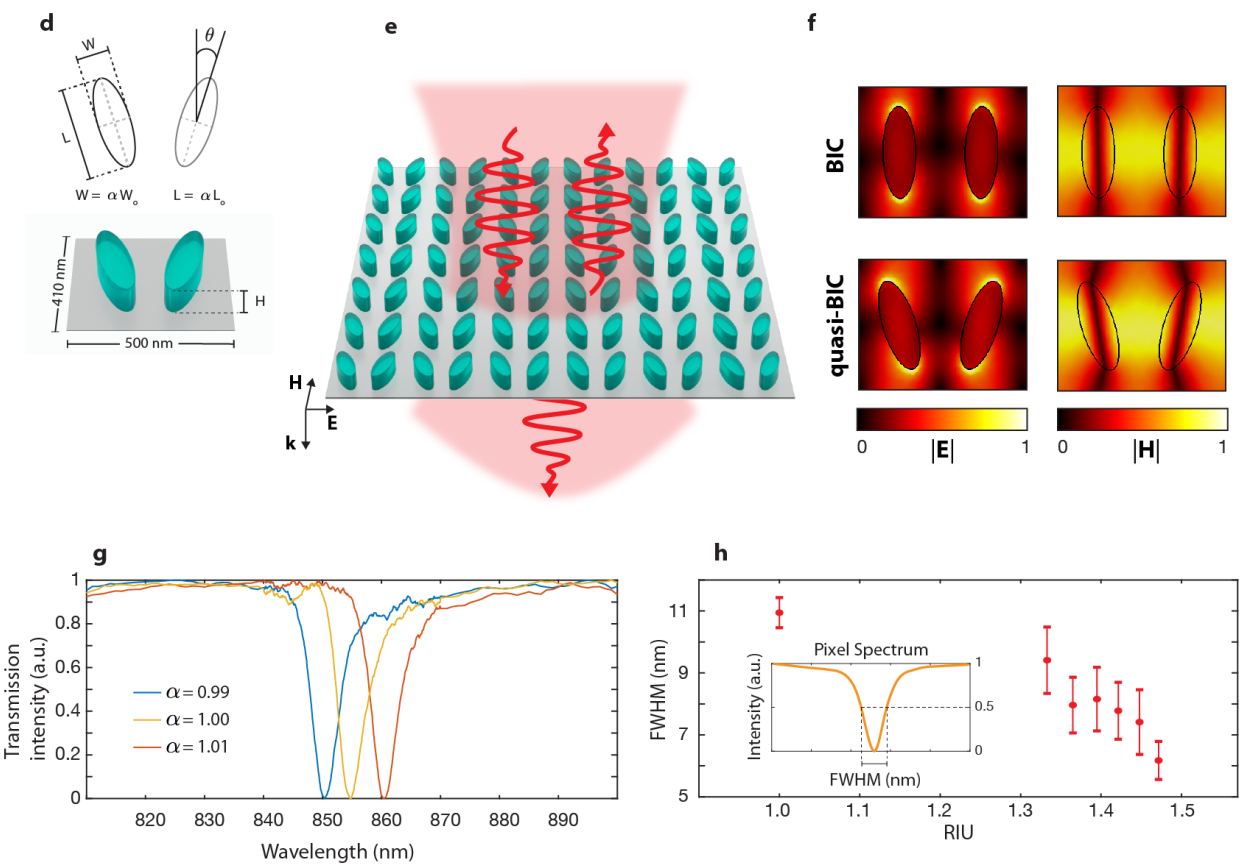

Figure 2 | Geometrically tunable high-Q dielectric metasurfaces based on quasi-BIC modes. Scanning electron microscope (SEM) images of the dielectric metaunit (a) and the metasurface (b). (c) Photograph of a dielectric chip with a $5 \times 5$ sensor array. (d) Top-view schematic and rendered image of the metaunit composed of two elliptical dielectric nanoresonators positioned in a mirror symmetry across the major vertical axis, where $\mathrm{W}_{\mathrm{o}}=100 \mathrm{~nm}, \mathrm{~L}_{\mathrm{o}}=280 \mathrm{~nm}, \mathrm{H}=100 \mathrm{~nm}$, and $\theta=17.5^{\circ}$. The parameter $\alpha$ is used to tune the resonance wavelength (see Fig. S4). (e) Schematic of light scattered by the metasurface. (f) Distribution of electric and magnetic fields in the metaunit showing both BIC and quasi BIC modes. (g) Transmission spectra extracted from the hyperspectral data cube from a single CMOS pixel sampled from sensor regions $\left(\sim 0.5 \mu \mathrm{m}^{2}\right)$ fabricated using three different scaling parameters $\left(\mathrm{n}_{\mathrm{M}}=1.47\right)$. The spectra exhibit narrow and spectrally isolated resonance dips in the near-IR. (h) Spectral linewidth of the resonance dip measured in the presence of top media with different refractive indices $\left(\mathrm{n}_{\mathrm{M}}=1-1.47\right)$ indicating Q-factors as high as 144 . 


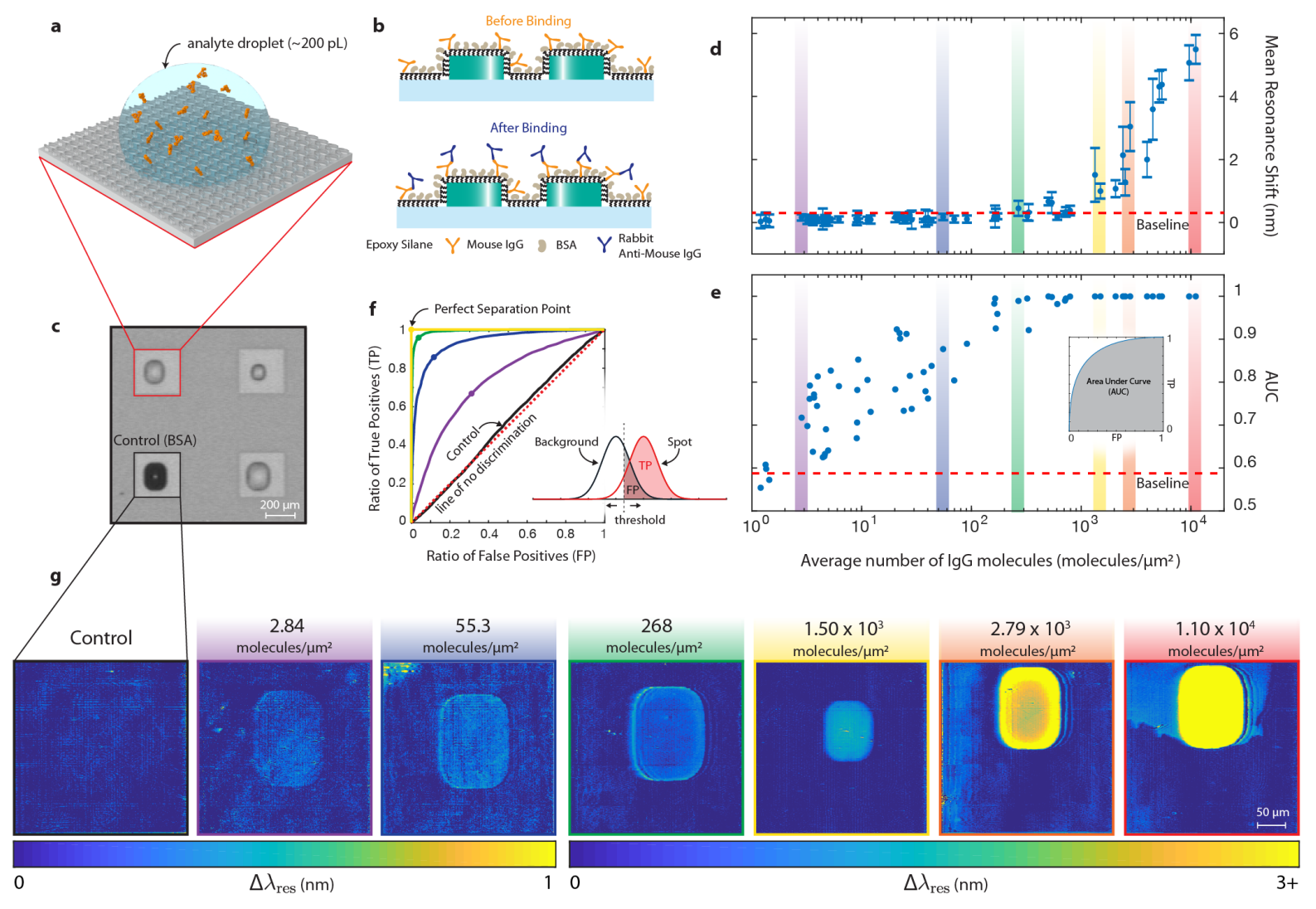

Figure 3 | Biosensing using dielectric metasurface sensors and image-based data processing. (a) Rendered schematic shows controlled biomolecule delivery to the sensor surface using low-volume ( $\sim 200 \mathrm{pL}$ ) droplets for microarray based detection. (b) Schematic of the bioassay, in which epoxy silane based covalent chemistry is used for biomolecule immobilization. (c) Image of a representative $2 \times 2$ sensor array showing analyte molecules dispensed using different volume droplets and a control sensor without analyte molecules. (d) A traditional calibration curve correlating ensemble-averaged resonance shift from binding spots to the average number of analyte molecules per area (molecules $/ \mu \mathrm{m}^{2}$ ). Note that the large standard deviation in the high concentration regime are caused by the coffee ring effect. (e) Area under the curve (AUC) values of all ROC curves. Control measurements revealed an AUC value of $0.5008 \pm 0.0291(\mathrm{~N}=68)$, and the baseline was set at 0.5881 . This approach shows that detection of biomolecules at low concentrations can be achieved, in contrast to traditional ensemble averaging. (f) Receiver operating characteristic (ROC) curve of a sample data set. (g) Resonance shift maps of a sample set from the data presented in (d) and (e) and (f). For other resonance shift maps at low molecule densities, see Fig. S9. 


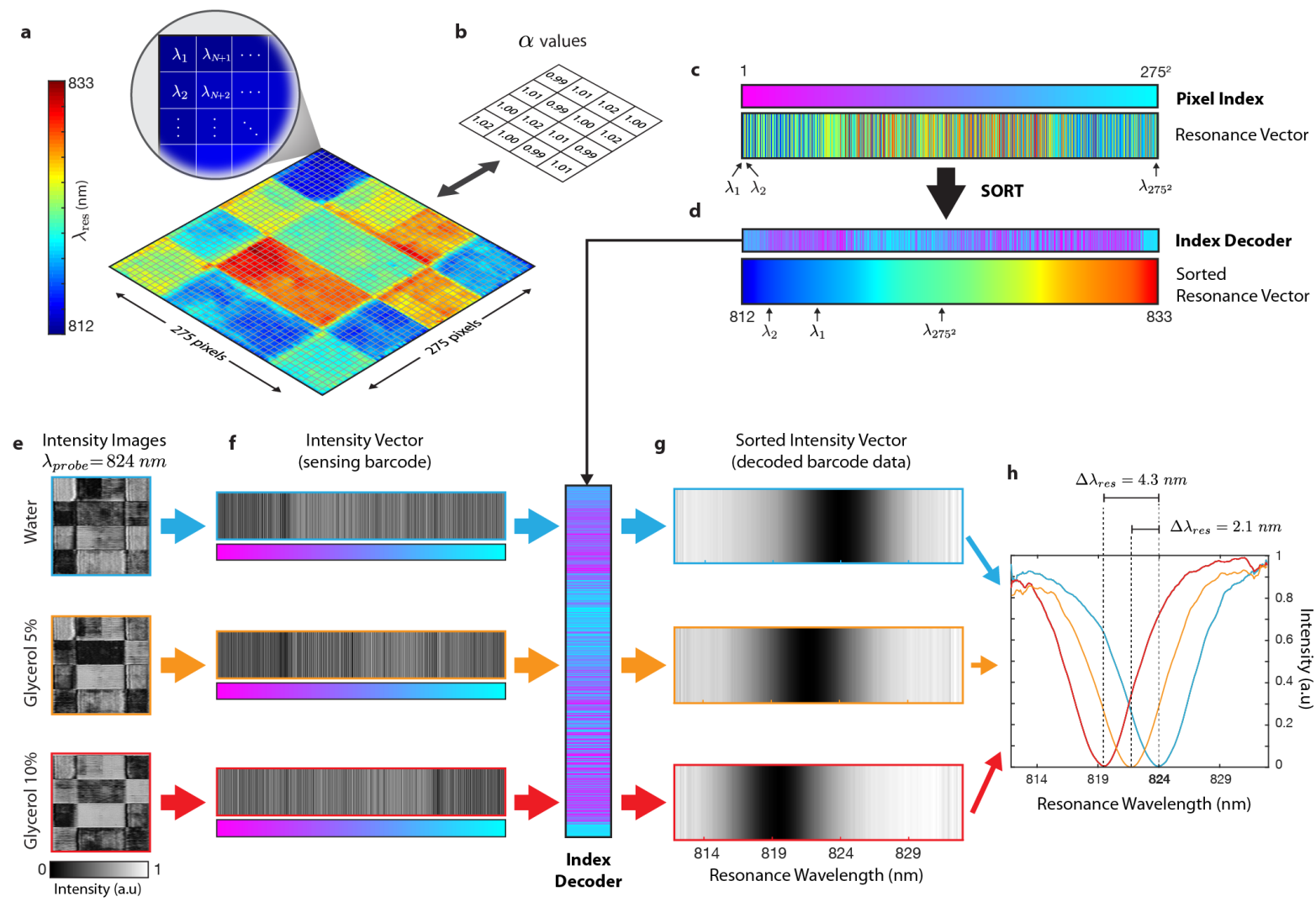

Figure 4 | Barcode-based sensing with multi-resonance dielectric metasurfaces. (a) Resonance map of a multi-resonance sensor, designed according to the scaling parameters in (b), recorded by $275 \times 275$ CMOS pixels. The distribution of resonance wavelengths within a sensor has a $20 \mathrm{~nm}$ spectral range. (c) Resonance vector made by stacking the resonance map column-wise. (d) Sorted resonance vector and its associated index decoder. (e) Intensity images of a multi-resonance sensor recorded at a single excitation wavelength $(824 \mathrm{~nm}$ ) with top medium of water, $5 \%$ and $10 \%$ glycerol. (f) Sensing barcodes created by stacking the individual pixel intensity values from the images in (e). (g) Intensity vectors sorted using the index decoder in (d), resulting in pseudo-spectra show in (h), where the difference in the wavelength of pixels that resonate at $824 \mathrm{~nm}$ corresponds to the spectral shift associated with the change in the refractive index of the top media. 


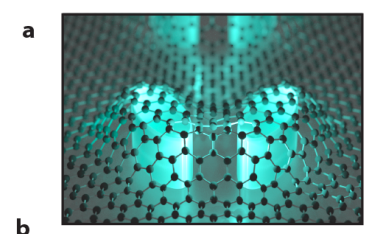

b

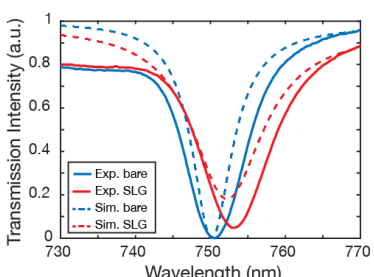

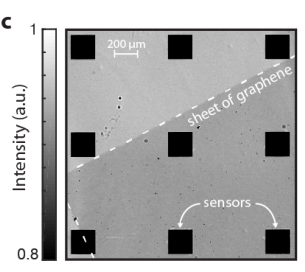

d

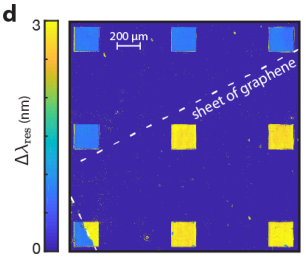

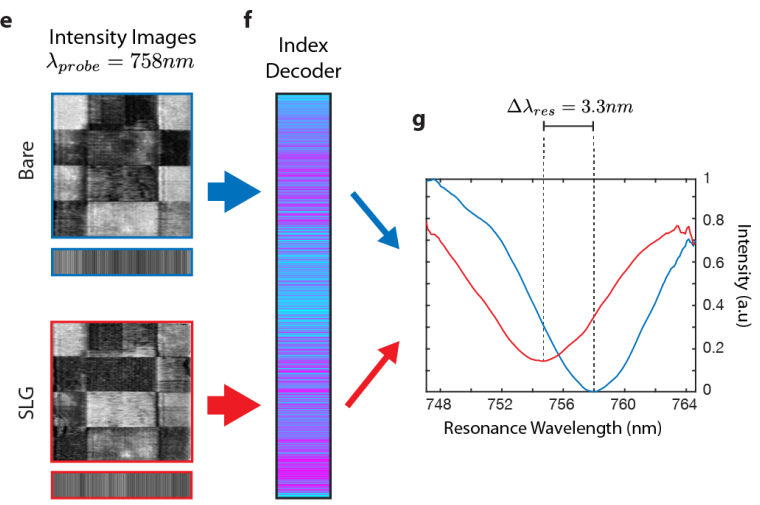

Figure 5 | Optical characterization of single atomic layer of graphene (SLG) using dielectric metasurface. (a) Rendered representative image of SLG covering dielectric nanoresonators. (b) Hyperspectrally measured and numerically calculated transmission spectra before and after the SLG transfer on the bare sensor extracted from a sub-sensor region. The resonance dip spectrally red-shifts and broadens due to graphene's optical properties in near-IR $(\mathrm{n}=2.69, \mathrm{k}=1.52)$ based on both simulation and experiment. (c) Large-area $\left(\sim 3.3 \mathrm{~mm}^{2}\right)$ image of a sensor array partially covered with a sheet of SLG, whose edge is marked by white dashed-lines and (d) its corresponding resonance shift map extracted from the hyperspectral data cubes before and after SLG transfer. (e) Intensity images of a multi-resonance sensor recorded at single excitation wavelength $(758 \mathrm{~nm})$ before and after SLG transfer and corresponding sensing barcodes. (f) Index decoder computed from the hyperspectral data cube collected from the bare sensor. The index decoder is used to sort the sensing barcodes in (e), resulting in the plots shown in (g). The spectral shift due to SLG is equivalent to the difference in the wavelengths of pixels that resonate at $758 \mathrm{~nm}$. 


\section{Methods}

All-dielectric metasurface fabrication. The all-dielectric metasurfaces were fabricated using $100-\mathrm{keV}$ electron beam lithography (EBL) by nanopatterning a thin layer $(100 \mathrm{~nm})$ of amorphous silicon (a-Si), which was deposited using low pressure chemical vapor deposition (LPCVD) on a clean bare fused silica substrate. First, a-Si coated wafers were diced with a photoresist protective layer into $1.5 \mathrm{~cm}$ X $1.5 \mathrm{~cm}$ chips and then individual chips were cleaned using resist remover and oxygen plasma. A double layer of positive tone EBL resist, Poly methyl methacrylate (PMMA 495K bottom and 950K top) was spin coated to yield $100 \mathrm{~nm}$ total thickness and baked at $180 \mathrm{C}$ for 5 mins. Double layer PMMA is crucial to create an undercut in the resist layer for successful lift-off process. Since the dielectric metasurfaces were fabricated on non-conductive fused silica substrates, $5 \mathrm{~nm}$ of thin Au layer was sputtered on the PMMA as a charge dissipation layer. After EBL exposure, first the Au film was wet etched, and then the EBL resist was developed. To transfer the pattern into the a-Si, aluminium hard mask (30 nm) was deposited using an e-beam evaporator. The metal lift-off was performed by soaking the chips in a resist remover bath at $70^{\circ} \mathrm{C}$ for 6 hours. The mask pattern was transferred into the a-Si film by fluorine-based inductively coupled plasma etching. Finally, the Al hard mask was wet-etched to complete the all-dielectric metasurface fabrication (See Fig. S1).

Graphene transfer and Raman spectroscopy characterization. For graphene transfer, CVD-grown graphene was wet-transferred over the clean dielectric metasurface sensor arrays. To certify the presence of a single layer of graphene, Raman spectroscopy was taken using inViaTM Raman microscope (Renishaw, Gloucestershire, UK) both on the nanostructured metasurface and on non-patterned glass area with excitation wavelengths of $532 \mathrm{~nm}$ and 785 nm (See Fig. S12 and Table S1).

Optical characterization set-up. The all-dielectric metasurfaces were optically characterized using a hyperspectral imaging-based analysis method. To acquire the hyperspectral data cube, individual images were recorded at narrow-band illumination using a CMOS camera (DS Qi2, Nikon, Tokyo, Japan), coupled to the outlet of an inverted microscope (Eclipse-Ti, Nikon, Tokyo, Japan). The illumination source frequency was swept using a Laser Line Tunable Filter (LLTF, NKT Photonics, Denmark) coupled to a supercontinuum laser source (SuperK EXTREME EXR-15, NKT Photonics, Denmark). The LLTF's operation is based on volume holographic gratings and is capable of tuning wavelengths $(\lambda)$ from $400 \mathrm{~nm}$ to $1000 \mathrm{~nm}$. The bandwidth (FWHM) of filter output is about $1.75 \mathrm{~nm}$ at $\lambda=700 \mathrm{~nm}$ with maximum FWHM of $2.5 \mathrm{~nm}$ at $\lambda=1000 \mathrm{~nm}$. The continuously tunable filter can suppress out of band transmission efficiently (60 dB $@ \pm 40 \mathrm{~nm}$ ) enabling a widely tunable narrow band illumination. A nonuniform spectral image sampling was used, where $\Delta \lambda=0.1 \mathrm{~nm}$ steps were used to resolve the sharp resonance and $\Delta \lambda=1 \mathrm{~nm}$ was used off the resonance to acquire a wide spectral range. To corroborate the hyperspectral data, the all-dielectric surfaces were also spectrally characterized using an imaging spectrometer and a high-performance CCD camera (IsoPlane 320 spectrometer, Pixis camera, Princeton Instruments, NJ, USA). The spectrometer is directly coupled to the outlet of an inverted microscope. The transmission spectra of the dielectric resonators were recorded using a dense grating $(600 \mathrm{gr} / \mathrm{mm})$ with blaze wavelength of $750 \mathrm{~nm}$ 
under normal broadband illumination and examined for the resonant dip properties (see Fig. S5).

Data acquisition and pre-processing. For each experiment, the hyperspectral cube from a sensor array was partitioned into smaller subsets, each containing the data cube of a single sensor, and saved as datasets of an .h5 file. Before using the data cube, the images were corrected for spatial and spectral variations of the light source. This was done by dividing each pixel value to the corresponding pixel value of a data cube collected with the same acquisition parameters, but without the sensor chip.

Resonance map computation. To build a resonance map, the spectrum associated with each image pixel is processed in MATLAB to extract the centre wavelength of its resonance. To obtain accurate values, data points from a window of three $\mathrm{nm}$ around the wavelength that contains the darkest pixel are used to fit a second-degree polynomial, whose minima is calculated and assigned to the pixel in question. The process is repeated for every pixel resulting in the resonance map.

Resonance shift map computation. The resonance shift map is calculated from two resonance maps from the same sensor taken at different time instances (for example, before and after analyte binding). An intensity image that contains high contrast between the sensor and offsensor areas is selected from both datasets. These images are used to compute the geometric transformation matrix that aligns them, where only rotation and translation are allowed. The geometric transformation matrix is used to align the previously computed resonance maps. The resonance shift map is the result of the element-wise subtraction of the two aligned resonance maps.

Full-wave numerical analysis. The numerical analysis of the all-dielectric metasurface is performed using a commercially available finite-element frequency-domain solver (CST Microwave Studio 2017). The presented transmittance and field distribution results are produced using periodic boundary conditions for each metaunit where a polarized $\left(\mathrm{TM}_{00}\right)$ incident light is launched from the top port at normal incidence. The geometric parameters are specified in the main text and the a-Si thin-film material parameters area shown in Fig. S2d. The largest mesh element dimension is kept below one tenth of the smallest wavelength used.

Surface chemistry and chip cleaning. A covalent surface chemistry based on (3glycidoxypropyl) trimethoxysilane (3-GPS) (Sigma Aldrich, MO, USA) monolayers was used to immobilize the capture antibody microarrays on all-dielectric metasurface. A continuous monolayer of 3-GPS forms on the dielectric metasurface when the silane end binds to the silicon oxide. The functional terminal group of 3-GPS is epoxide and it reacts with the amine groups of antibodies, which are used as capture molecules in our biorecognition assay. To form the self-assembled monolayer of 3-GPS, the chips were cleaned in Radio Corporation of America (RCA) first-step solution at $50^{\circ} \mathrm{C}$ for 30 mins. The clean chips were incubated in 3GPS solution in toluene $(1 \% \mathrm{v} / \mathrm{v})$ for $20 \mathrm{~min}$ and rinsed in fresh toluene to remove unbound 3GPS molecules. Then, the chips were dried using $\mathrm{N}_{2}$ gas and baked at $120^{\circ} \mathrm{C}$ for $30 \mathrm{~min}$. The epoxy-silane coated chips were shelf-stored under vacuum to be used within one month after preparation. The all-dielectric metasurfaces were used for multiple biosensing experiments. In 
between the bio experiments, the chips were cleaned using piranha solution $\left(\mathrm{NH}_{4} \mathrm{OH}: \mathrm{H}_{2} \mathrm{O}_{2}\right.$, 3:1) and then the above procedure was repeated. Note that in most of the low concentration measurements data was collected exclusively from fresh chips to eliminate any possible effect of chip recycling.

Bioassay details. The biorecognition assays were performed based on immunoassays (see Fig. S7a). For the sample sensors, primary capture antibodies (Mouse IgG ab37355, Abcam) were covalently immobilized in a microarray format on the epoxy-silane coated all-dielectric metasurfaces. A non-contact, low-volume liquid dispenser (sciFLEXARRAYER S3, Scienion, Dortmund, Germany) was used to create microarrays of antibody droplets ( $\sim 200 \mathrm{pL})$ on the sensors. Antibody solutions at various concentrations were prepared in phosphate buffered saline (PBS 1x) buffer with $0.5 \% \mathrm{w} / \mathrm{v}$ Trehalose and $0.01 \% \mathrm{w} / \mathrm{v}$ Tween. In order to control the total quantity of molecules in a microspot, various number of droplets were deposited and incubated approximately for $1 \mathrm{~h}$ at room temperature (RT). For control sensors, the bioassay steps are repeated except that, instead of $\mathrm{M}-\mathrm{IgG}$, bovine serum albumin (BSA, $1 \% \mathrm{w} / \mathrm{v}$ ) was spotted on the sensor. For blocking the rest of the surface, the chips were directly soaked in BSA ( $1 \% \mathrm{w} / \mathrm{v}$ in PBS $1 \mathrm{x}$ buffer) for 30 mins and then washed in PBS (1x) two times for 5 mins, rinsed in milli-Q water and dried. Next, the secondary antibody (Rabbit anti-mouse IgG ab6725, Abcam) solution ( $200 \mu \mathrm{g} / \mathrm{mL}$ in PBS 1x) is incubated over the microarray for $1 \mathrm{~h}$ at RT and then washed in PBS (1x) two times for 5 mins, rinsed in milli-Q water and dried.

Analyte quantity estimation. The number of mouse $\mathrm{IgG}$ molecules per metasurface area is estimated as follows. First, the total number of molecules in a microspot is calculated based on the total volume of dispensed IgG solution and its concentration. For instance, in a single droplet $(\sim 200 \mathrm{pL})$ of $2 \mu \mathrm{g} / \mathrm{mL} \operatorname{IgG}(150 \mathrm{kDa})$ solution, there are $1.6 \times 10^{6} \mathrm{IgG}$ molecules. Then, the total spot area is calculated using the images acquired. A single droplet microspot area usually covers approximately $1 \times 10^{4} \mu \mathrm{m}^{2}$ of the sensor area, which comprises $3.69 \times 10^{4}$ metaunits or $1.9 \times 10^{4}$ image pixels with a $10 \mathrm{X}$ objective. Assuming that every single IgG molecule dispensed in a droplet binds to the metasurface, the number of molecules per area, metaunit, or pixel can be estimated. For the above numerical example, this translates into 160 molecules $/ \mu \mathrm{m}^{2}, 43$ molecules/metaunit or 84 molecules/image pixel. Since our optical platform can resolve sensing information from a single image pixel, the molar correspondence of above example translates to as 0.14 zepto moles. Note that the molecular density of a given experiment depends on several experimental and environmental parameters, such as the spot volume, sampling variations, surface hydrophobicity (contributing to the spot wetting), humidity in the incubation chamber, and structural imperfections in sensor fabrication.

Thresholding and receiver operating characteristic curve. To quantify the presence of an analyte at low concentrations, a threshold method was implemented (See Fig. S7b). For each resonance shift map, two $75 \times 75$ subset of pixels were extracted: one from the region where the analyte was spotted and one from the region without analytes. The two sets of 5625 pixels were labelled positive and negative, respectively. The pixels were then classified using a standard threshold method, where pixels whose associated resonance shifts fall above the threshold $t$ are classified as positive, and the remaining pixels are classified as negative. The ratio of true positives $\left(R T P_{t}\right)$ was computed by dividing the number of pixels correctly classified as positive 
to the total number of pixels labelled positive, whereas the ratio of false positives $\left(R F P_{t}\right)$ was computed by dividing the number of pixels incorrectly classified as positive to the total number of pixels labeled negative. The process was repeated for different thresholds, and a receiver operating characteristic curve was built, where each point represents a threshold. To quantify the presence of an analyte, we chose the area under the ROC curve (AUC) as metric. ${ }^{23}$ For control measurements, data was extracted from control areas, either from control-specific sensors or off-spot regions in sample sensors.

\section{Data availability}

The data that support the plots within this paper and other findings of this study are available from the corresponding author upon reasonable request.

\section{Code availability}

The custom codes used in this study are available from the corresponding author upon reasonable request. 